This item was submitted to Loughborough's Research Repository by the author.

Items in Figshare are protected by copyright, with all rights reserved, unless otherwise indicated.

\title{
Nonlinear magnetization of graphene
}

PLEASE CITE THE PUBLISHED VERSION

http://dx.doi.org/10.1103/PhysRevB.86.125440

PUBLISHER

(c) The American Physical Society

VERSION

VoR (Version of Record)

LICENCE

CC BY-NC-ND 4.0

REPOSITORY RECORD

Slizovskiy, Sergey, and Joseph J. Betouras. 2019. "Nonlinear Magnetization of Graphene". figshare. https://hdl.handle.net/2134/12771. 


\title{
Nonlinear magnetization of graphene
}

\author{
Sergey Slizovskiy ${ }^{*}$ and Joseph J. Betouras ${ }^{\dagger}$ \\ Department of Physics, Loughborough University, Loughborough LE11 3TU, United Kingdom \\ (Received 30 March 2012; revised manuscript received 13 June 2012; published 24 September 2012)
}

\begin{abstract}
We compute the magnetization of graphene in a magnetic field, taking into account for generality the possibility of a mass gap. We concentrate on the physical regime where quantum oscillations are not observed due to the effect of the temperature or disorder and show that the magnetization exhibits nonlinear behavior as a function of the applied field, reflecting the strong nonanalyticity of the two-dimensional effective action of Dirac electrons. The necessary values of the magnetic field to observe this nonlinearity vary from a few teslas for very clean suspended samples to 20-30 T for good samples on substrate. In the light of these calculations, we discuss the effects of disorder and interactions as well as the experimental conditions under which the predictions can be observed.
\end{abstract}

DOI: 10.1103/PhysRevB.86.125440

PACS number(s): 75.70.Ak, 73.22.Pr

\section{INTRODUCTION}

The physics of graphene has attracted a huge amount of interest since its discovery ${ }^{1}$ due to its unique physical properties as well as its potential technological importance. The electronic properties and its behavior in a strong magnetic field have been the focus of wide activity as recent reviews summarize. ${ }^{2,3}$

The Dirac-like spectrum of clean graphene has been established; see, e.g., Ref. 4 and references therein. Moreover, the partition function of two-dimensional massless systems is known to exhibit strong nonanalytic behavior as a function of external fields. 5 ,6 This kind of behavior can lead, for example, to non-Ohmic conductivity due to Schwinger pair production at certain conditions ${ }^{7,8}$ or signatures of quantum criticality. ${ }^{9}$ In the case of conventional metals, the magnetism is a result of two contributions, coming from the spin (Pauli contribution) or the orbitals (Landau diamagnetism). In this work, we examine in detail the orbital magnetization of graphene in a magnetic field, including for generality the possibility of a mass gap, and show the appearance of a nonlinear dependence on the applied field, as a consequence of nonquadratic field dependence of the partition function. The Pauli magnetization of graphene is linear as a function of doping and much smaller than the orbital magnetization especially for low carrier densities (chemical potential close to 0 ). ${ }^{10}$ The possible nonlinearity due to the Pauli contribution, which is a known phenomenon, a consequence of the saturation of localized moments at higher fields, is discussed in the part of this work where the experimental verification is proposed.

The nonlinearity happens as a result of the failure of the linear response approximation, which is applicable when there is a mass scale larger than the applied perturbation. This scale controls the perturbative expansion. For the case of graphene this could be, e.g., a mass gap, the temperature, or the impurity scattering rate. Since the magnetic energy scale in graphene is known to be exceptionally large due its linear dispersion, even at moderate magnetic fields the magnetic energy scale becomes dominant, and therefore it violates the basic assumptions of the linear response regime.

The nonlinearity survives even at relatively small magnetic field for sufficiently clean samples; therefore it is not sufficient to compute only the zero-field magnetic susceptibility.
Typically, the observed nonlinear effect of strong magnetic field is the magnetic oscillations, but these oscillations average out at elevated temperatures or due to impurities producing the linear magnetization. Here we focus on the regime where the magnetic oscillations average out but, nevertheless, a smooth nonlinearity remains. In the following sections we discuss the effects of temperature, mass gap, disorder, as well as interaction effects at a phenomenological level and propose an experiment which can demonstrate these predictions.

\section{CLEAN GRAPHENE WITH POSSIBLE MASS GAP}

At zero temperature, the grand canonical potential of clean graphene in a magnetic field when Landau levels (LLs) are formed, at zero chemical potential, with energy of the LLs $E_{n}=\operatorname{sign}(n) \sqrt{\alpha|n B|}$ where $\alpha=2 \hbar e v_{F}^{2}$ and $n$ is an integer, reads

$$
\Omega_{\mathrm{vac}}=g_{s} g_{v} \sqrt{\alpha}|B|^{3 / 2} C\left(\frac{\zeta(3 / 2)}{4 \pi}-0.1654 a \sqrt{|B| C}\right)
$$

with $a=0.142 \mathrm{~nm}$ being the distance between the carbon atoms and the degeneracy factor $C=\frac{e}{2 \pi \hbar}, g_{v}=2, g_{s}=2$ accounts for spin and valley degeneracy, $\zeta$ is the zeta function: $\frac{\zeta(3 / 2)}{4 \pi}=-\zeta(-1 / 2) \approx 0.2079$.

The subleading terms are lattice corrections which are discussed elsewhere; ${ }^{10,11}$ the numerical factor corresponds to nearest-neighbor tight-binding model calculation and describes orbital paramagnetic contributions due to higher energy levels having non-Dirac dispersion. The corrections as a result of the deviation of the dispersion from the Dirac-like appear either due to interaction effects-which we discuss below-or due to lattice effects when $B \gg 100 \mathrm{~T}$ and are not discussed further here.

The leading term, though, is nonanalytic in the magnetic field and naturally leads to divergent diamagnetic susceptibility at the Dirac point. Such nonanalyticities are typical not only in the effective action of massless systems but at quantum critical points ${ }^{12-15}$ or even in corrections to Fermi liquid theory. ${ }^{16}$

When temperature-averaged over many Landau levels (LLs), this nonanalytic contribution cancels out, as first shown by McClure. ${ }^{17}$ When the magnetic field overcomes the energy scale set up by the temperature (or impurity scattering), this 
nonlinearity can be observed. For generality and to compare energy scales, we take into account a mass gap $\Delta$ in the dispersion relation, which can be experimentally created, e.g., due to A-B sublattice asymmetry caused by SiC substrate or by regular deposition of impurities. ${ }^{20,21}$ At zero magnetic field it was demonstrated that the pseudospin degree of freedom (due to valleys) produces diamagnetic susceptibility which is ${ }^{22-24}$

$$
\chi(\epsilon)=-g_{s} g_{v} \frac{\alpha C}{12|\Delta|} \theta(|\Delta|-\epsilon) .
$$

It is evident that the mass gap resolves the formal $\delta$-function singularity in the susceptibility, but when the magnetic energy scale exceeds that of the gap, the nonanalyticities of the free energy become again dominant, as we will show.

In a magnetic field $B$ and in the presence of the mass gap, the spectrum at low energies becomes

$$
E_{n \neq 0}=\operatorname{sign}(n) \sqrt{\alpha|n B|+\Delta^{2}}, \quad E_{0}=(-1)^{v} \Delta,
$$

where $v=0,1$ enumerates the two Dirac valleys. In the absence of the gap or when its size is smaller than the distance between the first LLs $\Delta<\sqrt{\alpha|B|}$, the linear expression (1) is not applicable and, as we will show, the correct result leads to the nonlinear magnetization.

The regularized free energy reads (at chemical potential $\mu=0$, temperature $T=0$ )

$$
\begin{aligned}
& \Omega(\Delta, 0,0)=-g_{s} C|B|\left(\sum_{n=0}^{\infty}+\sum_{n=1}^{\infty}\right) \sqrt{\alpha n|B|+\Delta^{2}} \\
& \stackrel{\text { reg }}{=}-g_{s} C \alpha^{1 / 2}|B|^{3 / 2}\left[-\left(\frac{\Delta^{2}}{\alpha|B|}\right)^{1 / 2}\right. \\
& \left.+2 \zeta\left(-\frac{1}{2}, \frac{\Delta^{2}}{\alpha|B|}\right)+\frac{4}{3}\left(\frac{\Delta^{2}}{\alpha|B|}\right)^{3 / 2}\right] \text {, }
\end{aligned}
$$

where we have regularized and subtracted the $B=0$ expression; $\zeta$ is a generalized Hurwitz $\zeta$ function. It also agrees with related formula in Ref. 22. The above expression is exact in the limit of free electrons and has a strong-field expansion valid for $\delta^{2} \equiv \frac{\Delta^{2}}{\alpha|B|} \lesssim 1$ (when the gap is less than or comparable to the distance between the first LLs), leading to the strong-field expansion of the magnetization $(\mu=0, T=0)$ :

$$
\begin{aligned}
M(\Delta, 0,0)= & g_{s} C \sqrt{\alpha|B|}\left[3 \zeta(-1 / 2)+\delta+\frac{\zeta(1 / 2)}{2} \delta^{2}\right. \\
& \left.+\frac{\zeta(3 / 2)}{8} \delta^{4}-\frac{3 \zeta(5 / 2)}{16} \delta^{6}+\mathcal{O}\left(\delta^{8}\right)\right],
\end{aligned}
$$

where $\delta \equiv \sqrt{\frac{\Delta^{2}}{\alpha|B|}}=27.5 \frac{\Delta(\mathrm{eV})}{\sqrt{|B|(\mathrm{T})}}$; for example, for $\Delta=$ $0.1 \mathrm{eV}$ this formula works starting from approximately $7.5 \mathrm{~T}$, indicating the scale from which the nonlinearities occur.

At $T=0$ and as $\Delta \rightarrow 0$ the range of the values of $B$ where linear magnetization occurs decreases to zero. Therefore, the nonlinearity of magnetization gets stronger with the reduction of the gap and temperature. The susceptibility, following from
Eq. (3), reads

$$
\begin{aligned}
\chi(\Delta, 0,0)= & \frac{g_{s} C \sqrt{\alpha}}{2 \sqrt{|B|}}\left[3 \zeta\left(-\frac{1}{2}, \frac{\Delta^{2}}{\alpha|B|}\right)\right. \\
& \left.-2 \frac{\Delta^{2}}{\alpha|B|} \zeta\left(\frac{1}{2}, \frac{\Delta^{2}}{\alpha|B|}\right)-\frac{\Delta^{4}}{\alpha^{2}|B|^{2}} \zeta\left(\frac{3}{2}, \frac{\Delta^{2}}{\alpha|B|}\right)\right] .
\end{aligned}
$$

At finite temperature and chemical potential, after performing the same subtraction as for the regularization of $\Omega(\Delta, \mu=0, T=0)$, and additionally subtracting $\mu N(\mu=$ $0, T=0)$ which is independent of $B$, we obtain ${ }^{25}$

$$
\begin{aligned}
& \Omega(\Delta, \mu, T) \\
& =\Omega(\Delta, 0,0)-g_{s} C B k T\left[\ln \left(1+\exp \left(\frac{-\Delta+\mu}{k_{B} T}\right)\right)\right. \\
& \left.\quad+2 \sum_{n=1}^{\infty} \ln \left(1+\exp \left(\frac{-E_{n}+\mu}{k_{B} T}\right)\right)\right]-[\mu \rightarrow-\mu] .
\end{aligned}
$$

At zero chemical potential the temperature plays a role similar to the gap. It is instructive to note that if we compare McClure's result for magnetic susceptibility at finite temperature,

$$
\chi=-\frac{g_{s} g_{v} C}{24} \frac{\alpha}{k_{B} T} \operatorname{sech}^{2} \frac{\mu}{2 k_{B} T},
$$

with the one for the magnetization with gap but at zero temperature, we see that at $2 k_{B} T=\Delta$ the linear magnetization is the same (with $\mu=0$ ), while the nonlinear parts are different as is shown in Fig. 1.

For nonzero chemical potential and temperature there are two regimes, the well-known low-field regime and the high-field regime which sets in when $E_{1}=\sqrt{\alpha|B|} \gtrsim 2 \mu$. When the chemical potential exceeds the temperature scale, the magnetization rapidly decreases as expected, but it starts to grow when the separation between the LLs becomes comparable to the chemical potential; this behavior is shown

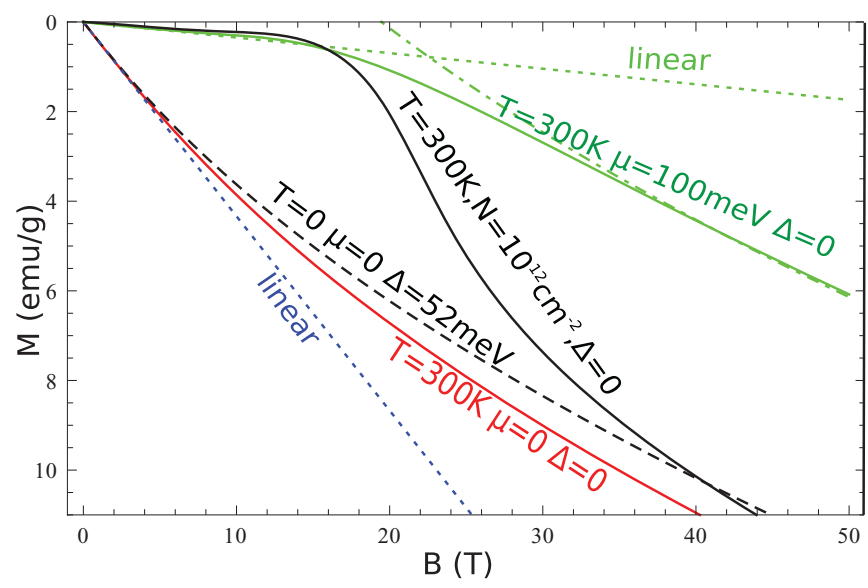

FIG. 1. (Color online) The magnetization at $T=300 \mathrm{~K}, \mu=0$ and at gap values $\Delta=0$ and $52 \mathrm{meV}$ is compared to the linear behavior. In addition, the magnetization at fixed chemical potential $100 \mathrm{meV}$ and at fixed electron concentration $10^{12} \mathrm{~cm}^{-2}$ at $T=$ $300 \mathrm{~K}$ is plotted. Low and high field asymptotic are shown. 
by the upper curve in Fig. 1. At such fields the zeroth LL gives the leading constant paramagnetic contribution to the magnetization, but the same nonlinear vacuum energy contribution remains: For $\sqrt{\alpha|B|} \gg 2(\mu+\Delta) \gg k_{B} T$,

$$
M(\Delta, \mu, T) \approx g_{s} C(\mu-\Delta)+M(\Delta, 0,0) .
$$

This asymptotic regime is shown by the upper green dotdashed line in Fig. 1. When the temperature decreases we get de Haas-van Alphen oscillations for nonzero chemical potential, as expected. ${ }^{22}$ The non-linearity we discuss can be alternatively interpreted as being connected to these $\mathrm{dHvA}$ oscillations, as its remnant behavior.

When the number of particles is fixed, instead of the chemical potential (both situations are experimentally realizable in cases of graphene on substrate or suspended flakes) then $\mu$ is expressed through the relation $N=-\frac{\partial \Omega}{\partial \mu}$. At small temperature, the de Haas-van Alphen oscillations are observed. At high fields the chemical potential inevitably tends to zero since almost all the electrons (or holes if $\mu<0$ ) can be hosted by the zero LL. This leads to the $|B|^{1 / 2}$ asymptotic behavior of magnetization, corresponding to Eq. (3), shown by the black solid curve in Fig. 1 .

For completeness, we briefly comment on the case of bilayer graphene where the zero-field susceptibility ${ }^{26,27}$ diverges logarithmically with the Fermi energy $\epsilon_{F} \rightarrow 0$ and the divergence is cut by the greatest of trigonal warping scale $\epsilon_{\text {trig }}$ and $\epsilon_{F}$. When we increase the magnetic field, the magnetic energy scale $\sqrt{\alpha|B|}$ eventually becomes the greatest, thus leading to weak logarithmic nonlinearity of the magnetization. The asymptotic form of the magnetic thermodynamic potential is $^{28} \Omega=\frac{g_{s} g_{v}}{8 \pi} \frac{e^{2} v_{F}^{2}}{\gamma_{1}} \ln \left[\gamma_{1} /(\sqrt{\alpha|B|})\right] B^{2}$, where $\gamma_{1} \approx 0.4 \mathrm{eV}$ is the interlayer hopping energy and the magnetic scale $\sqrt{\alpha|B|}$ is assumed to be larger than the trigonal warping energy $\epsilon_{\text {trig }}$ and $\epsilon_{F}$; otherwise one replaces $\sqrt{\alpha|B|} \rightarrow \epsilon_{F}$. Then one gets

$$
M \approx-|B| \frac{g_{s} g_{v} e^{2} v_{F}^{2}}{8 \pi \gamma_{1}} \ln \left(\frac{\gamma_{1}^{2}}{|B| \alpha}\right) .
$$

At even larger magnetic fields $B \gtrsim 100 \mathrm{~T}$, the magnetic energy becomes larger than the interlayer hopping $\gamma_{1}$, thus effectively reducing the bilayer to two monolayers. Since the nonlinearity of magnetization of the graphene bilayer is significantly weaker than for the monolayer, we expect that the impurities would make this effect hard to observe. So, we concentrate on the monolayer in what follows. The $N$-layered graphene was shown to have [N/2] bilayer bands and $N \bmod$ 2 monolayer bands. ${ }^{26}$

\section{EFFECT OF IMPURITIES}

Besides the mass gap and temperature, the nonlinearity of the magnetization is influenced by impurities and interactions. We consider first the short-range scattering impurities and then the effect of charge inhomogeneities: the electron and hole puddles.

\section{A. Short-range impurities}

Consider for simplicity the short-range impurities with momentum-independent scattering. It is sufficient to adopt the self-consistent Born approximation (SCBA). ${ }^{29,31}$ The treatment of the vacancy-type impurities ${ }^{32}$ or the commonly used phenomenological Lorentzian broadening leads to similar conclusions qualitatively, as we have checked.

In $\mathrm{SCBA},{ }^{29}$ the self-energy reads

$$
\Sigma(\epsilon)=\frac{W \alpha|B|}{2} \sum_{n} \frac{g\left(\epsilon_{n}\right)}{\epsilon-\epsilon_{n}-\Sigma(\epsilon)},
$$

where $\epsilon_{n}$ is a full spectrum and $g(\epsilon)$ is some smooth cutoff function. This is valid for small strength of disorder $W=$ $\frac{n_{i} u_{i}^{2}}{4 \pi v_{F}^{2}} \ll 1$, where $n_{i}$ is the impurity concentration and $u_{i}$ a measure of the strength of the random on-site impurity interaction. For a discrete LL spectrum and for small $W$ we obtain the solution iteratively. The density of states is

$$
\rho(\epsilon)=-\frac{g_{v} g_{s}}{2 \pi^{2} \hbar^{2} v_{F}^{2} W} \operatorname{Im} \Sigma(\epsilon+i 0) .
$$

For the strong-field regime the single-level approximation of the level width works well, as has been checked numerically. Solving Eq. (9) with a single energy level we obtain two roots and the relevant solution is $\Sigma(\epsilon)=\frac{1}{2}\left[\epsilon-\epsilon_{n}-\operatorname{sign}(\epsilon-\right.$ $\left.\left.\epsilon_{n}\right) \sqrt{\left(\epsilon-\epsilon_{n}\right)^{2}-2 \alpha W B}\right]$; therefore the density of states can be approximated by a semicircle form

$$
\rho(\epsilon)=\frac{g_{v} g_{s} C}{\pi \alpha W} \sqrt{2 \alpha W|B|-\epsilon^{2}} \theta\left(2 \alpha W|B|-\epsilon^{2}\right),
$$

where $\theta$ is a step function and $\epsilon$ means the deviation from the LL of the clean system. In the single-level approximation, we neglect the shift of the level center due to the real part of the self-energy. Similar form for the density of states is supported by more elaborate computations; see, e.g., Ref. 30. Note that the level width scales as $\sqrt{|B|}$. The above approximation can be applied for levels $\epsilon_{n}$ up to $n \lesssim \frac{1}{8 W}$; for higher levels their overlap would become essential. Since at medium or strong fields the higher levels are typically far from the Fermi surface, we may use the same level-broadening for all the levels, since this does not alter the result for the magnetization.

Under the assumption that all the levels are broadened with the same profile $\rho(\epsilon)$, the partition function can be computed by integrating Eq. (6) with the broadened chemical potential:

$$
\Omega_{\mathrm{imp}}=\int d \epsilon \frac{\rho(\epsilon-\mu)}{\int \rho^{2}\left(\epsilon^{\prime}\right) d \epsilon^{\prime}} \Omega(\Delta, \epsilon, T) .
$$

We note that this equation automatically inherits the regularization from $\Omega(\Delta, \epsilon, T)$. For the magnetization one gets a similar formula, but with an extra contribution coming from the $B$ derivative of the broadening profile. At high fields this contribution is paramagnetic due to broadening of the zero LL. As mentioned above, there is no significant dependence on the actual width of higher LLs. The effect of temperature may be alternatively taken into account, by convolving the impurity broadening with the derivative of the Fermi function with respect to energy $f^{\prime} .^{33}$

The typical linewidth of the high-quality sample st,37 $^{3}$ estimated to be $\delta \approx 3 \sqrt{B(\mathrm{~T})} \mathrm{meV}$, so $W \approx 0.003$ and the Fermi energy is $\mu \approx 14 \mathrm{meV}$. Such high-quality sample is close to the ideal case and exhibits strongly nonlinear $\sim \sqrt{|B|}$ magnetization already at $1 \mathrm{~T}$, as illustrated in Fig. 2 .

From this subsection we conclude that low concentrations of short-range impurities do not significantly alter the 


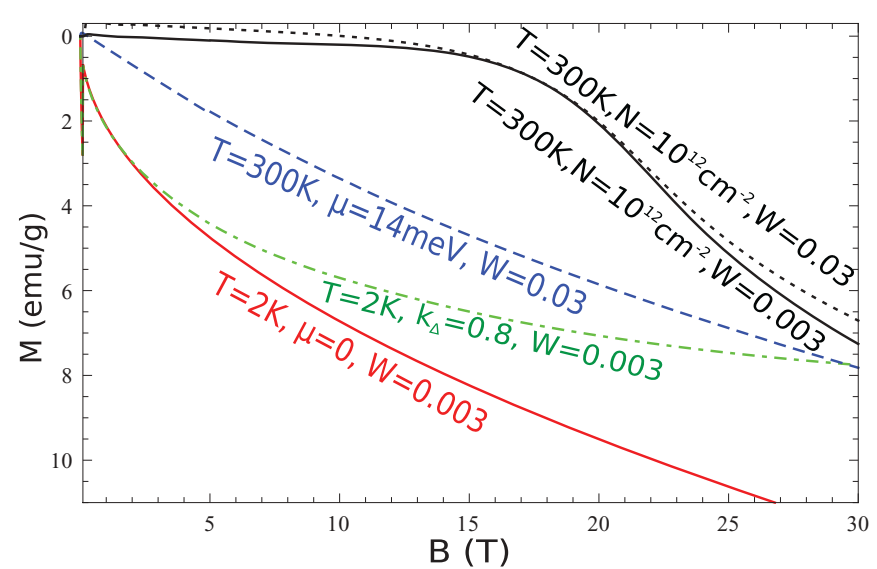

FIG. 2. (Color online) Nonlinear magnetization with short-range impurities for fixed $\mu$ or for fixed $N$. Green dashed curve takes into account the linear in $B$ splitting of zero LL due to interactions, while the green dot-dashed curve is for $\sim \sqrt{B}$ splitting of zero LL; see Sec. IV.

magnetization. For larger concentrations of impurities the above analysis is not applicable.

\section{B. Charge inhomogeneities}

It was shown experimentally that for many graphene samples the charge inhomogeneities play the dominant role. ${ }^{38}$ The effect of charge inhomogeneities can be modeled as longrange smooth variation of carrier density $N$ (electron and hole puddles), and the range may be assumed to be comparable to the size of cyclotron orbits. A simple model proposed in Ref. 38 fits well the experiment. It assumes Gaussian variation of $N$ with standard deviation of order $\delta N \approx 4 \times 10^{11} \mathrm{~cm}^{-2}$. Since $N \sim \mu^{2}$, the variation $\delta N$ leads to large $\delta \mu$ close to the tip of the Dirac cone and results in the large broadening of the zeroth LL. This contrasts with the equal profile broadening, coming from the scattering on short-range impurities. Apart from variation of the charge density, the charged impurities cause a usual broadening of the levels. We use the simple model of constant Lorentzian broadening, independent of the magnetic field, as compared to short-ranged impurities, where we got the $\sqrt{B}$ dependence. This does not significantly change the result and is partly justified by computations in Ref. 35, where the $B$ dependence was shown to be more shallow than $\sqrt{B}$ due to the fact that the screening increases with $B$.

To perform calculations, we broaden the levels with Lorentz profile, getting the density of states $\rho_{\epsilon}(E)$ then integrate it to find $N\left(E_{F}\right)$ and its inverse $E_{F}(N)$, and then contract with Gaussian density fluctuation profile:

$$
P(N, \bar{N}, \delta N)=\frac{1}{\sqrt{2 \pi} \delta N} \exp \left(-\frac{(N-\bar{N})^{2}}{2 \delta N^{2}}\right) .
$$

For example, the density of states is given by

$$
\rho(\bar{N})=\int d N \rho_{\epsilon}\left(E_{F}(N)\right) P(N, \bar{N}, \delta N) .
$$

The consideration of graphene with fixed total number of electrons is equivalent to the situation of graphene on substrate and the particle number being proportional to gate voltage. For

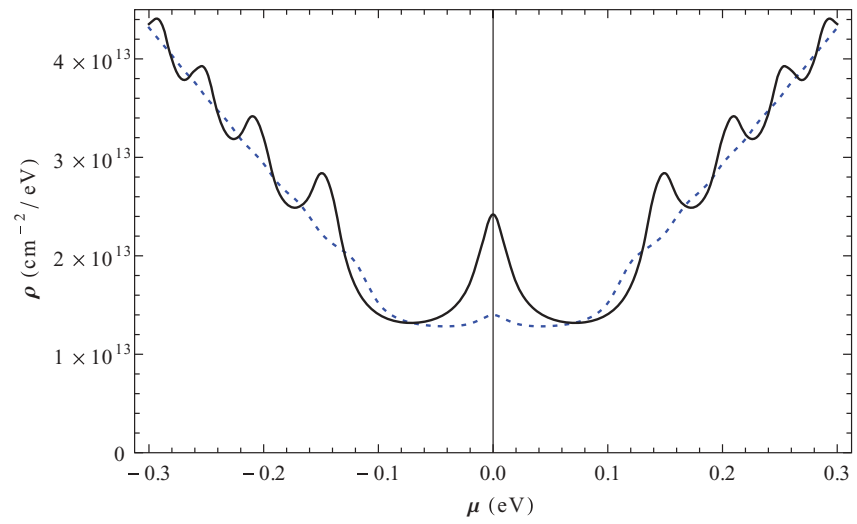

FIG. 3. (Color online) Density of states for $16 \mathrm{~T}$ (solid) and $10 \mathrm{~T}$ (dotted) as a function of chemical potential of neutral spot with Gaussian carrier density fluctuation $\delta N \approx 4 \times 10^{11} \mathrm{~cm}^{-2}$ and Lorentzian broadening of levels with half-width $\epsilon=15 \mathrm{meV}$.

suspended graphene (or exfoliated flake) one may imagine a situation of the fixed local chemical potential at spots where it touches a contact. Assuming that these spots are far from the charged impurity (neutral spot), the local electron density at such spots would coincide with the average value $\bar{N}$, so we may use the same as above function $N\left(E_{F}\right)$ to convert $\rho(\bar{N})$ to $\rho(N(\mu))$. The resulting density of states (DOS) as a function of the chemical potential is plotted in Fig. 3 for magnetic fields of 10 and $16 \mathrm{~T}, \delta N \approx 4 \times 10^{11} \mathrm{~cm}^{-2}$, and Lorentzian broadening with half-width $\epsilon=15 \mathrm{meV}$. The same figure but plotted against the electron density $N$ (or gate voltage) can be found in Ref. 38. For high temperatures, the level broadening is dominated by temperature, and for low temperatures, by impurities.

For low temperatures and Lorentz impurity broadening $\epsilon$ Eq. (6) for the grand canonical potential combined with Eq. (12) gives at $T=0$

$$
\begin{aligned}
\Omega(\Delta, \mu, \epsilon)= & \Omega(\Delta, 0,0) \\
& +g_{s} C B\left[F(\mu+\Delta)+2 \sum_{n=1}^{\infty} F\left(\mu+E_{n}\right)\right] \\
& +[\mu \rightarrow-\mu],
\end{aligned}
$$

where $E_{n}=\sqrt{\alpha n|B|+\Delta^{2}}$,

$$
\begin{aligned}
F(e)= & \frac{e}{\pi}\{\arctan [\max (\Lambda, e) / \epsilon]-\arctan [\max (-\Lambda, e) / \epsilon]\} \\
& +\frac{\epsilon}{2 \pi} \ln \frac{\max (e,-\Lambda)^{2}+\epsilon^{2}}{\max (e, \Lambda)^{2}+\epsilon^{2}}
\end{aligned}
$$

and $\Lambda$ is a large cutoff for Lorentzian broadening.

To see the effect of charge puddles on magnetization, we compute the magnetization as a function of electron density $N$ and then convolve with Gaussian profile:

$$
M(\bar{N}, \delta N, T)=\frac{1}{\sqrt{2 \pi} \delta N} \int d N M(N, T) e^{-\frac{(\bar{N}-N)^{2}}{2(\delta N)^{2}}} .
$$

This prescription follows from summation over separate puddles; $\bar{N}$ denotes the average electron density.

The results for fixed average carrier number $\bar{N}$ and for fixed chemical potential $\mu$ of neutral spot are shown in Figs. 4 and 5. From these plots we see that charge disorder observed in 


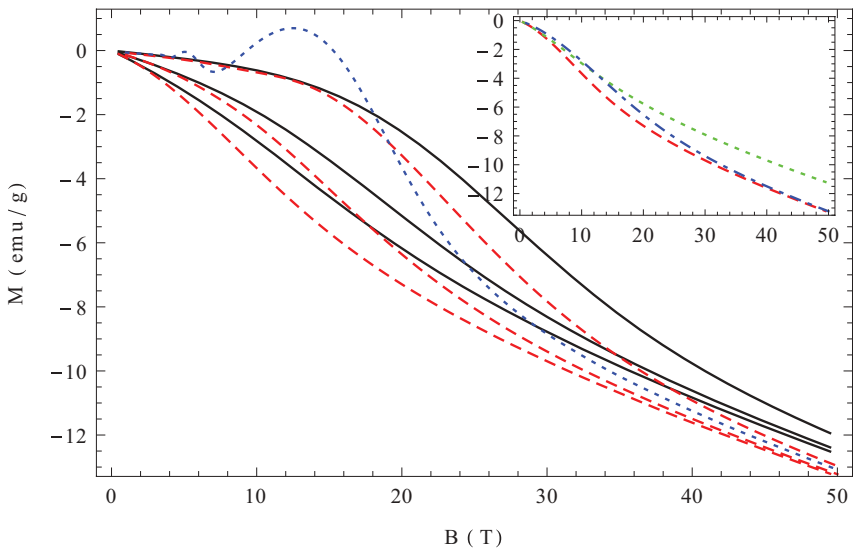

FIG. 4. (Color online) Nonlinear magnetization with charged impurities for fixed average electron density. Bottom to top: $\bar{N}=$ $0,5 \times 10^{11}, 10^{12}, 1.5 \times 10^{12} \mathrm{~cm}^{-2}$; temperature $T=300 \mathrm{~K}$ (black solid) and $T=0$ (red dashed); Lorentz broadening $\epsilon=15 \mathrm{meV}$; density fluctuation dispersion is $\delta N=4 \times 10^{11} \mathrm{~cm}^{-2}$. A plot for smaller $\delta N=4 \times 10^{10} \mathrm{~cm}^{-2}$ with $\bar{N}=10^{12} \mathrm{~cm}^{-2}$ and $T=0$ (blue dotted) is shown for comparison. Inset: Dependence on the impurity strength. Red dashed curve is the same as the bottom one on the main plot: $\bar{N}=0, T=0, \epsilon=15 \mathrm{meV}, \delta N=4 \times 10^{11} \mathrm{~cm}^{-2}$; green dotted: Lorentz broadening increased to $\epsilon=30 \mathrm{meV}$; blue dot-dashed: density fluctuation increased to $\delta N=6 \times 10^{11} \mathrm{~cm}^{-2}$.

experiments plays an important role in smearing the magnetic oscillations (the plot for lower charged disorder shows clear magnetic oscillations), as does also the temperature, but the resulting magnetization is still nonlinear. The nonlinearity of magnetization gets weaker with increase of both types of disorder, but in a different way; see the insets to Figs. 4 and 5.

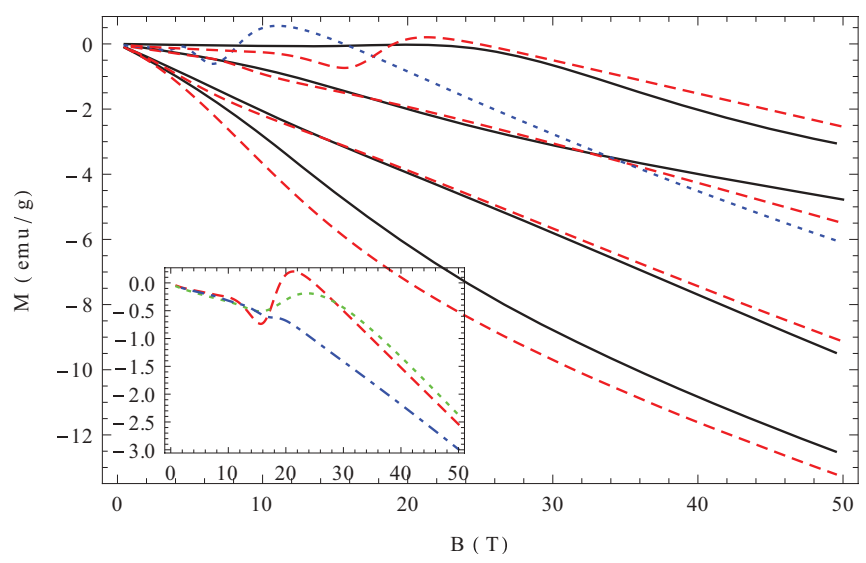

FIG. 5. (Color online) Nonlinear magnetization charged impurities for fixed chemical potential of neutral spot. Bottom to top: $\mu=0,50,100,150 \mathrm{meV}$; temperature $T=300 \mathrm{~K}$ (black solid) and $T=0$ (red dashed); Lorentz broadening is $\epsilon=15 \mathrm{meV}$; density fluctuation dispersion is $\delta N=4 \times 10^{11} \mathrm{~cm}^{-2}$. A plot for smaller $\delta N=4 \times 10^{10} \mathrm{~cm}^{-2}, \mu=100 \mathrm{meV}, T=0$ (blue dotted) is shown for comparison. Inset: Dependence on the impurity strength. Red dashed curve is the same as on the main plot: $\mu=150 \mathrm{meV}$, $T=0, \epsilon=15 \mathrm{meV}, \delta N=4 \times 10^{11} \mathrm{~cm}^{-2}$; green dotted: Lorentz broadening increased to $\epsilon=30 \mathrm{meV}$; blue dot-dashed: density fluctuation increased to $\delta N=6 \times 10^{11} \mathrm{~cm}^{-2}$.

\section{EFFECT OF INTERACTIONS}

It is worth noting that the most drastic effect of interactions is the splitting of the zero LL into two levels separated by a new gap $2 \tilde{\Delta}$ (different from the initial $\Delta$ ), when $\mu \approx 0$ and the zero LL is not completely filled. ${ }^{36,37,39} \tilde{\Delta}$ grows with magnetic field. The precise form of its dependence on the magnetic field is an open question and depends on the sample. Generally, there are the following kinds of energy gaps as a consequence of the magnetic field: (i) linear in $B$ dependence, coming from the Zeeman spin splitting $\Delta_{Z}=2 \mu_{B} B \approx 0.11 B(\mathrm{~T}) \mathrm{meV}$ and from the potential pseudospin splitting (from Kekulétype distortion of the lattice) $\Delta_{\text {Kekule }} \approx 0.2 B(\mathrm{~T}) \mathrm{meV}$, and (ii) interaction contributions, scaling as $\sqrt{B}$, as explained, e.g., in Ref. 3 and references therein.

The experimental data in Ref. 37 contain significant uncertainty, allowing for various fits. For samples with high mobility $17000 \mathrm{~cm}^{2} /(\mathrm{V} \mathrm{s})$ the fit by $\sqrt{B}$ looks reasonable: $\tilde{\Delta} \approx k_{1 / 2} \sqrt{|B|}$, with $k_{1 / 2} \approx 3 \mathrm{meV} / \sqrt{\mathrm{T}}$. For samples with lower mobility a fit linear in $B$ is within the error bars: $\tilde{\Delta} \approx k_{\text {linear }}|B|$ with $k_{\text {linear }} \approx 0.8 \mathrm{meV} / \mathrm{T}$ at half-filling. This is 7 times larger than the Zeeman splitting, indicating a different mechanism.

The splitting of the zeroth LL leads to an extra paramagnetic contribution, as compared to Eq. (6) with $\tilde{\Delta}=0$, $\mu=0$. The correction to thermodynamic potential is easily computed by replacing $\ln 2 \rightarrow \frac{1}{2} \ln \left(1+e^{-\tilde{\Delta} /\left(k_{B} T\right)}\right)+\frac{1}{2} \ln (1+$ $\left.e^{+\tilde{\Delta} /\left(k_{B} T\right)}\right)$. For the linear dependence on $B$, defining $x=$ $k_{\text {linear }}|B| / k_{B} T$ we have in the single-particle approximation:

$$
\delta M=C g_{s} k_{B} T\left(2 \ln \cosh \frac{x}{2}+x \tanh \frac{x}{2}\right) .
$$

This is shown by the dot-dashed curve on Fig. 2. The paramagnetic nature of this contribution can be understood as due to the reduction in energy of the filled half of the zeroth LL by interactions. The correction is quadratic for small $x$ and linear at large $x$.

For $\sqrt{B}$ splitting, the correction of the magnetization is

$$
\delta M=C g_{s} k_{B} T\left(2 \ln \cosh \frac{x}{2}+\frac{1}{2} x \tanh \frac{x}{2}\right)
$$

with $x=\frac{k_{1 / 2} \sqrt{|B|}}{k_{B} T}$.

The different fittings of the splitting of the zeroth LL as a function of $B$ lead to different $B$ dependence of the magnetization, which, in turn, can provide one more method to distinguish between the main possibilities. We note that still there is no fully satisfactory theoretical explanation of the level-splitting effect, ${ }^{37}$ and its microscopic explanation is beyond the scope of this work.

\section{EXPERIMENTAL PROPOSAL AND DISCUSSION}

The measurements of nonlinear magnetization can be used to study various properties of a graphene sample: Magnetization is sensitive to the number of carriers, mass gap, and disorder as well as the number of layers. In particular, one can extract the magnetic field dependence of the interactioninduced splitting of the zero LL. One possible way to measure the nonlinear magnetization is to measure the magnetization of 
a suspended graphene flake with scanning SQUID microscopy. Alternatively, one may go to higher magnetic fields (up to $50 \mathrm{~T}$ ) with a larger amount of lower quality graphene samples, e.g., the graphene laminate, used in Ref. 40. At high fields the cyclotron orbits would fit better in the small-sized flakes and one can neglect the boundary effects. In real samples there will be a significant nonlinear part of the magnetization, coming from localized impurity spins (Pauli contribution), but this effect can be fitted at lower magnetic fields or fields parallel to the surface, and consistently subtracted. ${ }^{41}$ Moreover, at magnetic fields greater than or of the order of $10 \mathrm{~T}$ and at low temperatures $T<4 \mathrm{~K}$, it is expected that all the localized moments will come to saturation; therefore the detected nonlinearity will be a consequence of the orbital contribution.

In conclusion, the two-dimensional nature and the linear spectrum of graphene are the necessary conditions to observe nonlinear magnetization at accessible magnetic fields. The underlying reason is the breakdown of the linear response theory due to the fact that the magnetic energy is the dominant in the system. The linear dispersion relation gives relatively large distances between the LLs and the two-dimensionality leads to absence of $k_{z}$ dispersion of LLs. We have found that even at room temperature and with moderate concentration of impurities the nonlinearity should be revealed at about 10-20 T, while with very clean suspended samples at low temperatures a lower value of magnetic field is sufficient. There are two types of nonlinear behavior that are present: Near half filling, the magnetization scales as $\sqrt{B}$ at higher fields, due to nonanalyticity of the effective action; then at higher values of the chemical potential, the magnetization is small and linear at small fields, while it increases the slope after the first LL crosses the Fermi energy, and at even higher magnetic fields one can also observe the $\sqrt{B}$ behavior.

\section{ACKNOWLEDGMENTS}

We acknowledge inspiring discussions with Feo Kusmartsev, Marat Gaifullin, and Roberto Soldati and discussions of experimental results with Irina Grigorieva and Paulina Plochocka. This work was supported by the Engineering and Physical Sciences Research Council under EP/H049797/1.
*On leave from PNPI; s.slizovskiy@lboro.ac.uk

$\dagger$ j.betouras@lboro.ac.uk

${ }^{1}$ K. S. Novoselov, A. K. Geim, S. V. Morozov, D. Jiang, Y. Zhang, S. V. Dubonos, I. V. Grigorieva, and A. A. Firsov, Science 306, 666 (2004).

${ }^{2}$ A. H. Castro Neto, F. Guinea, N. M. Peres, K. S. Novoselov, and A. K. Geim, Rev. Mod. Phys. 81, 109 (2009).

${ }^{3}$ M. O. Goerbig, Rev. Mod. Phys. 83, 1193 (2011).

${ }^{4}$ B. Partoens and F. M. Peeters, Phys. Rev. B 74, 075404 (2006).

${ }^{5}$ A. N. Redlich, Phys. Rev. D 29, 2366 (1984).

${ }^{6}$ D. Cangemi and G. Dunne, Ann. Phys. 249, 582 (1996).

${ }^{7}$ D. Allor, T. D. Cohen, and D. A. McGady, Phys. Rev. D 78, 096009 (2008).

${ }^{8}$ B. Dóra and R. Moessner, Phys. Rev. B 81, 165431 (2010).

${ }^{9}$ M. Müller, J. Schmalian, and L. Fritz, Phys. Rev. Lett. 103, 025301 (2009).

${ }^{10}$ G. Gómez-Santos and T. Stauber, Phys. Rev. Lett. 106, 045504 (2011).

${ }^{11}$ S. Slizovskiy and J. J. Betouras (unpublished).

${ }^{12}$ D. Belitz, T. R. Kirkpatrick, and T. Vojta, Phys. Rev. B 55, 9452 (1997).

${ }^{13}$ D. Belitz, T. R. Kirkpatrick, A. J. Millis, and T. Vojta, Phys. Rev. B 58, 14155 (1998).

${ }^{14}$ A. V. Chubukov and D. L. Maslov, Phys. Rev. B 68, 155113 (2003).

${ }^{15}$ D. V. Efremov, J. J. Betouras, and A. Chubukov, Phys. Rev. B 77, 220401 (2008).

${ }^{16}$ J. Betouras, D. Efremov, and A. Chubukov, Phys. Rev. B 72, 115112 (2005).

${ }^{17}$ J. W. McClure, Phys. Rev. 104, 666 (1956).

${ }^{18}$ S. Y. Zhou, G.-H. Gweon, A. V. Fedorov, P. N. First, W. A. de Heer, D.-H. Lee, F. Guinea, A. H. Castro Neto, and A. Lanzara, Nat. Mater. 6, 916 (2007).

${ }^{19}$ S. Y. Zhou, D. A. Siegel, A. V. Feodorov, F. El Gabaly, A. K. Schmid, A. H. Castro Neto, D.-H. Lee, and A. Lanzara, Nat. Mater. 7, 259 (2008).

${ }^{20}$ H. Sahin and S. Ciraci, Phys. Rev. B 84, 035452 (2011).
${ }^{21} \mathrm{~A}$ band gap of order $0.1 \mathrm{eV}$ has been observed in graphene epitaxially grown on $\mathrm{SiC}$ substrate (Refs. 18 and 19), but a word of caution is needed here, since substrate may induce high-impurity concentrations.

${ }^{22}$ S. G. Sharapov, V. P. Gusynin, and H. Beck, Phys. Rev. B 69, 075104 (2004).

${ }^{23}$ M. Nakamura, Phys. Rev. B 76, 113301 (2007).

${ }^{24}$ M. Koshino and T. Ando, Phys. Rev. B 81, 195431 (2010).

${ }^{25}$ In the context of dynamical flavor symmetry breaking in $2+1$ relativistic field theory models, related work can be found in V. P. Gusynin, V. A. Miransky, and I. A. Shovkovy, Phys. Rev. D 52, 4718 (1995); K. G. Klimenko, Z. Phys. C 54, 323 (1992); Theor. Math. Phys. 90, 1 (1992).

${ }^{26}$ M. Koshino and T. Ando, Phys. Rev. B 76, 085425 (2007).

${ }^{27}$ S. A. Safran, Phys. Rev. B 30, 421 (1984).

${ }^{28}$ M. I. Katsnelson and G. E. Volovik, JETP Lett. 95, 411 (2012).

${ }^{29}$ M. Koshino and T. Ando, Phys. Rev. B 75, 235333 (2007).

${ }^{30}$ B. Dóra, Low Temp. Phys. 34, 801 (2008).

${ }^{31}$ N. Shon and T. Ando, J. Phys. Soc. Jpn. 67, 2421 (1998).

${ }^{32}$ N. M. R. Peres, F. Guinea, and A. H. Castro Neto, Phys. Rev. B 73, 125411 (2006).

${ }^{33}$ Equation (12) can be straightforwardly generalized to the case when the zero LL has a different broadening profile $\rho_{0}$ by adding $\int_{-\infty}^{\mu} d \epsilon \epsilon\left[\rho_{0}(\epsilon)-\rho(\epsilon)\right]$ where the profiles need to be convoluted with the derivative of the Fermi function $f^{\prime}$ to account for temperature.

${ }^{34}$ M. L. Sadowski, G. Martinez, M. Potemski, C. Berger, and W. A. de Heer, Phys. Rev. Lett. 97, 266405 (2006).

${ }^{35}$ C. H. Yang, F. M. Peeters, and W. Xu, Phys. Rev. B 82, 205428 (2010).

${ }^{36}$ Y. Zhang, Z. Jiang, J. P. Small, M. S. Purewal, Y.-W. Tan, M. Fazlollahi, J. D. Chudow, J. A. Jaszczak, H. L. Stormer, and P. Kim, Phys. Rev. Lett. 96, 136806 (2006).

${ }^{37}$ E. A. Henriksen, P. Cadden-Zimansky, Z. Jiang, Z. Q. Li, L.-C. Tung, M. E. Schwartz, M. Takita, Y.-J. Wang, P. Kim, and H. L. Stormer, Phys. Rev. Lett. 104, 067404 (2010). 
${ }^{38}$ L. A. Ponomarenko, R. Yang, R. V. Gorbachev, P. Blake, A. S. Mayorov, K. S. Novoselov, M. I. Katsnelson, and A. K. Geim, Phys. Rev. Lett. 105, 136801 (2010).

${ }^{39}$ A. J. M. Giesbers, L. A. Ponomarenko, K. S. Novoselov, A. K. Geim, M. I. Katsnelson, J. C. Maan, and U. Zeitler, Phys. Rev. B 80, 201403 (2009).
${ }^{40}$ M. Sepioni, R. R. Nair, S. Rablen, J. Narayanan, F. Tuna, R. Winpenny, A. K. Geim, and I. V. Grigorieva, Phys. Rev. Lett. 105, 207205 (2010).

${ }^{41}$ R. R. Nair, M. Sepioni, I-Ling Tsai, O. Lehtinen, J. Keinonen, A. V. Krasheninnikov, T. Thomson, A. K. Geim, and I. V. Grigorieva, Nat. Phys. 8, 199 (2012). 